May 19, 2010

\title{
ON EMBEDDING RINGS IN CLEAN RINGS
}

\author{
W.D. BURGESS AND R. RAPHAEL
}

\begin{abstract}
A clean ring is one in which every element is a sum of an idempotent and a unit. It is shown that every ring can be embedded in a clean ring as an essential ring extension. It is seen that the centre of a clean ring need not be a clean ring. There is no "clean hull" of a ring. A family of examples is given where there is a ring $R$, not a clean ring, embedded in a commutative clean ring $S$ so that there is no clean ring $T, R \subseteq T \subseteq S$, minimal with that property. It is also shown that a commutative pm ring cannot be extended to a clean ring by the adjunction of finitely many central idempotents.
\end{abstract}

1. Introduction. The notion of a clean ring was introduced by Nicholson ([23]) and has been studied extensively since. (See [5] for a sample of the references.) Clean rings are exchange rings and the two classes coincide when all idempotents are central ([23, Proposition 1.8]), but not in general (Camillo and $\mathrm{Yu}$, [6, page 4746]). The theme of this article is to examine the embedding of an arbitrary ring in a clean ring.

Notation and terminology. All rings in what follows are assumed to be rings with 1 . For a ring $R, \mathbf{U}(R)$ is the group of units, $\mathbf{J}(R)$ the Jacobson radical, $\mathbf{B}(R)$ the set and also boolean algebra of central idempotents and $\mathbf{P}(R)$ the prime radical. A ring $R$ is indecomposable if $\mathbf{B}(R)=\{0,1\}$. A local ring $R$ is one where $R / \mathbf{J}(R)$ is a division ring. If $R$ is a subring of a ring $S$ and $T$ is a subset of $S$, then the subring of $S$ generated by $R$ and $T$ is denoted $R[T]$. The term "regular ring" will mean "von Neumann regular ring". The notation of Lam $([18])$ is usually followed.

Definition 1.1. An element $r$ in a ring $R$ is called clean if it can be written $r=e+u$, where $e=e^{2}$ and $u$ is a unit in $R$. A ring $R$ is clean if all its elements are clean.

A ring $R$ is called 2-clean if every element in it can be written as a sum of an idempotent and two units. Wang and Chen ([26, Theorem 7])

1991 Mathematics Subject Classification. 16U99, 13A99, 54C35.

Key words and phrases. clean ring, rings of continuous functions, local ring.

The work of the second author was supported by the NSERC. 
have shown that every ring can be embedded in a 2-clean ring because the ring of $2 \times 2$ matrices over any ring is 2 -clean. It will be shown here that every ring can also be embedded in a clean ring, although not in such an explicit fashion as for 2-clean rings.

Indeed, every ring can be embedded in a clean ring as a ring essential extension (Theorem 2.1). Some more explicit embeddings are then looked at. In the process, it is observed (Proposition $2.5(2)$ ) that the centre of a clean ring is not necessarily clean. A characterization of clean rings which are algebras over commutative 0-dimensional rings is found using some central localizations (Proposition 2.8).

It is shown that, in some special cases, when a ring $R$, which is not clean is embedded in a clean ring $S$, there are clean rings between $R$ and $S$ which are minimal among such (Proposition 3.2). However, even when these minimal clean extensions in a given extension exist, they are not unique (except in a very special case, Proposition 3.8)) and, hence, there is no notion of a clean hull of a ring. Moreover, a natural family of examples is presented of rings $R$ embedded in clean rings $S$ for which, while there are clean rings strictly between $R$ and $S$, there are none which are minimal with that property (Theorem 3.6); these include all rings of continuous functions which are not already clean.

Every commutative clean ring is a pm ring (each prime ideal is contained in a unique maximal ideal, Anderson and Camillo, [1, Corollary 4]). Every commutative pm ring can be extended to a clean ring by the adjunction of central idempotents ([3, Theorem 3.12]). However, a commutative ring which is not a pm ring cannot be embedded in a clean ring by an integral extension ([3, Theorem 3.12]). It is shown (Theorem 4.3) that a commutative pm ring which is not clean cannot be made into a clean ring by adding finitely many central idempotents. (Theorem 4.3).

At various points in the sequel the language of the Pierce sheaf of a ring will be used (see [25] and [16, $\mathbf{V} 2]$ ). If $R$ is any ring the base space of the sheaf is Spec $\mathbf{B}(R)$, a boolean space (compact, Hausdorff and totally disconnected). The stalks are the rings $R_{x}=R / R x$, for $x \in \operatorname{Spec} \mathbf{B}(R)$ and a basis for the topology of the espace étalé is given by elements of $R$ over clopen (closed and open) subsets of $\operatorname{Spec} \mathbf{B}(R)$. Then $R$ is isomorphic to the ring of sections of this sheaf.

On the other hand if $\mathcal{R}$ is a sheaf of indecomposable rings over a boolean space $X$ with ring of sections $R$, the key point which makes $\mathcal{R}$ the Pierce sheaf of $R$ is that when two local sections coincide at some $x \in X$, they coincide over a neighbourhood of $x$ ([25, Definitions 3.1 and 4.1]). 
It is important to note that if all the idempotents of a ring $R$ are central then $R$ is a clean ring if and only if the stalks of its Pierce sheaf are local ([4, Proposition 1.2] or [16, V., Proposition 2.6]).

2. Various embeddings. The first theorem in this section is based on results from [5] by V. Camillo, et al. In that paper, a (left) $R$-module $M$ is called clean if $\operatorname{End}\left({ }_{R} M\right)$ is a clean ring ([5, page 96$\left.]\right)$.

It is first shown that every ring can be embedded in a clean ring.

Recall that a ring extension $R \subseteq S$ is called an essential ring extension if, for every ideal $\mathbf{0} \neq I \subseteq S, I \cap R \neq \mathbf{0}$.

Theorem 2.1. Every ring can be embedded as an essential ring extension in a clean ring.

Proof. It is first shown that a ring $R$ can be embedded into a clean ring. Let $R$ be any ring which will be considered, for now, as a $\mathbf{Z}-R$ bimodule. According to [5, Corollary 3.13], the endomorphism ring of a pure injective (= algebraically compact) module is clean. If $R^{\mathbf{N}}$ is the full product of countably many copies of $R$ and $\mathcal{F}$ is a non-principal ultrafilter on $\mathbf{N}$, put $I_{\mathcal{F}}$ to be the $\mathbf{Z}$-submodule

$$
I_{\mathcal{F}}=\left\{\left(r_{n}\right) \in R^{\mathbf{N}} \mid \text { for some } F \in \mathcal{F}, r_{n}=0 \forall n \in F\right\} .
$$

The subgroup $I_{\mathcal{F}}$ is also an ideal of the ring $R^{\mathbf{N}}$. According to $[15$, item 3, page 337] (see also Jensen and Zimmermann-Huisgen [17, Corollary E]) $S=R^{\mathbf{N}} / I_{\mathcal{F}}$ is $\mathbf{Z}$-pure injective. Moreover, $R$ embeds, as a ring, in the ring $S$. Since $R$, and hence, $S$ are rings with 1, right multiplication gives an embedding of $S$ into $T=\operatorname{End}(\mathbf{z} S)$, which is a clean ring.

In the above embedding, let $K$ be an ideal of $T$ maximal with respect to $K \cap R=\mathbf{0}$. Then, $C=R / K$ is, as a homomorphic image of a clean ring, an essential ring extension of $R$ which is clean.

Corollary 2.2. A simple (prime, semiprime, subdirectly irreducible) ring can be embedded in a simple (prime, semiprime, subdirectly irreducible, respectively) clean ring.

Proof. Using Theorem 2.1, it only needs to be observed that the properties listed are all preserved under essential ring extensions.

Remark 2.3. If $R$ is embedded in a clean ring $S$ then the subring $T$ of $S$ generated by $R$ and the units of $S$ is also clean. If 2 is invertible in $S$ then $T=S$.

Proof. Each $t \in T$ can be written $t=e+u$ in $S, e=e^{2}$ and $u$ a unit. Then, it follows that $e \in T$ as well. If 2 is invertible in $S$ then for 
$e=e^{2} \in S, 1-e / 2$ has inverse $1+e$ showing that $e \in T$ and, hence, $T=S$.

The question about embedding in clean rings has now been solved in a rather abstract way but special kinds of rings have embeddings which are of a more familiar sort, that is, more closely related to basic ring theory. There follows a list of some of these.

The commutative case has been dealt with in detail in $[3, \S 4.2$ and 4.3]. For any commutative ring $R$, here supposed not clean, $R$ embeds in the full product of local ring $\Pi=\prod_{P \in \operatorname{Spec} R} R_{P}$ since $R$ is a ring of sections of a sheaf of these local rings. Let $\mathbf{B}_{c}(R)$ be the boolean algebra of idempotents from $\Pi$ whose supports in $\operatorname{Spec} R$ are the subsets of Spec $R$ which are both closed and open in the constructible (patch) topology on $\operatorname{Spec} R$. Then, as shown in [3, Construction 4.6], the subring $\mathfrak{j} \mathfrak{c}(R)$ of $\Pi$ generated by the image of $R$ and $\mathbf{B}_{c}(R)$ is a clean ring. (In fact, the construction is functorial.)

Proposition 2.4. Let $R$ be commutative ring: (1) $R$ can be embedded in the commutative clean ring $\mathfrak{j} \mathfrak{c}(R)$. (2) $R$ can be embedded in the endomorphism ring of the injective hull $E=E\left({ }_{R} R\right)$.

Proof. (1) [3, Construction 4.6]. (2) Since $R$ is commutative, $Q_{\max }(R)$ is the centre of the endomorphism ring $H$ of $E$ ([18, Proposition 13.24]); $H$ is a clean ring.

In the case of $R=\mathbf{Z}$, the ring $\mathfrak{j c}(\mathbf{Z})$ is the ring of sequences in $\prod_{p \text { prime }} R_{(p)}$ which are eventually constant as elements of $\mathbf{Q}$.

It does not seem to have noticed been before that the centre of a clean ring is not necessarily clean.

Proposition 2.5. (1) A ring which is its own complete ring of quotients is not necessarily clean. (2) The centre of a clean ring is not necessarily clean.

Proof. (1) Let $S$ be a commutative ring which is not a clean ring and $M$ the direct sum of a copy of each simple $S$-module. The trivial extension $R=S \oplus M$ is a Kasch ring ([18, Proposition 8.30]) and, hence, its own complete ring of quotients. It is not a clean ring since $S$ is a homomorphic image. (2) As already seen (Proposition 2.4) if $R$ is commutative then $Q_{\max }(R)$ is the centre of a clean ring. Then it suffices to choose a Kasch ring, as in (1), which is not clean to get an example. Taking $S=\mathbf{Z}$ would suffice.

If a ring $R$ can be embedded in a regular $\operatorname{ring} S$ then it can be embedded in a clean regular ring, for example by the left or right 
maximal ring of quotients of $S$. Such rings have been characterized in [10, Theorem 1.4.4] as follows: $R$ can be embedded in a regular ring if and only if its additive group $R^{+}$has no elements of square order. This class includes all semiprime rings. However, semiprime rings can also be seen to be among those rings which can be embedded in a direct product of full endomorphism rings of vector spaces over fields. It suffices to embed a semiprime ring $R$ into a direct product of prime rings $R \subseteq \prod_{\alpha \in A} R_{\alpha}$ and then, via the extended centroid $K_{\alpha}$ of $R_{\alpha}$ ([18, Corollary 14.22]) into $\operatorname{End}\left(K_{\alpha} R_{\alpha}\right)$. The following is also clear.

Remark 2.6. (1) If a ring $R$ contains a division ring $D$ as a subring then $R$ embeds in the clean ring $\operatorname{End}\left({ }_{D} R\right)$. (2) If $R$ is left (right) nonsingular then its complete left (right) ring of quotients is clean.

There are cases where it is not necessary to use Theorem 2.1 but $R$ as a $\mathbf{Z}$-module is nevertheless employed.

Proposition 2.7. (1) If the additive group $R^{+}$of a ring $R$ is of bounded order then $\operatorname{End}(\mathbf{z} R)$ is clean. (2) If $R^{+}$can be written as $R^{+}=D \oplus E$, where $D$ is divisible and $\operatorname{ann}_{\mathbf{Z}} E=\mathbf{0}$, then $R$ embeds in $S=\operatorname{End}\left({ }_{\mathbf{z}} R\right)$ and $S$ is clean. (3) If $R^{+}$is torsion-free then $R$ embeds in $T=R \otimes_{\mathbf{z}} \mathbf{Q}$, which in turn embeds in the clean ring $\operatorname{End}(\mathbf{z} T)$.

Proof. (1) If 1 is of order $n$ in $R^{+}$, then $R^{+}$is a $\mathbf{Z} /\langle n\rangle$-module and $\mathbf{Z} /\langle n\rangle$ is a commutative artinian principal ideal ring. Then, [5, Examples $31.4(2)]$ says that $S=\operatorname{End}_{\mathbf{z} /\langle n\rangle}\left(R^{+}\right)$is a clean ring. However, $R$ embeds in $S$ via right multiplications.

(2) [15, page 344, item 3] says that $R^{+}$is $(\Sigma)$-pure injective, and, hence, $S$ is clean.

A subdirectly irreducible ring $R$ where $R^{+}$is a mixed group is of the type in Proposition 2.7 (2), by [9, Observation 4.4.3].

It is shown in [5, Examples 3.14(4)] that an extension of a field by certain central nilpotents is a clean ring. However, such a ring is commutative and zero-dimensional, i.e., is a $\pi$-regular ring; hence, it is a clean ring. Moreover, the following generalization is true. Recall that an element $r$ in a ring $R$ is called strongly clean if it can be written $r=e+u, e^{2}=e, u \in \mathbf{U}(R)$ and $e u=u e$; if all elements of $R$ are strongly clean, $R$ is called a strongly clean ring.

Proposition 2.8. Let $A$ be a commutative 0-dimensional ring and $R$ an A-algebra. Then, (i) $R$ is a (strongly) clean ring if and only if for each $\mathfrak{m} \in \operatorname{Max} A, R$ localized at $A \backslash \mathfrak{m}$ is a (strongly) clean ring; and (ii) if the module ${ }_{A} R$ is locally finitely generated then $R$ is a clean ring. 
Proof. (i) Let $R^{(\mathfrak{m})}$ be the localization of $R$ at $A \backslash \mathfrak{m}$. Assume first that, for each $\mathfrak{m} \in \operatorname{Max} A, R^{(\mathfrak{m})}$ is clean. In Goodearl and Warfield, [13, Proposition 2], it is seen how to lift solutions of finite sets of polynomial equations in each $R^{(\mathfrak{m})}$ to solutions in $R$. For any $r \in R$, consider the following set of polynomials: $r-y_{1}-y_{2}, y_{1}^{2}-y_{1}, y_{2} y_{3}-1$ and $y_{3} y_{2}-1$. By assumption, this set of polynomials has a solution in each $R^{(\mathfrak{m})}$, giving a global solution $r_{1}, r_{2}, r_{3} \in R$ showing that $r$ is a clean element.

In the other direction it is assumed that $R$ is a clean ring. For any $a \in A$ there is $e \in \mathbf{B}(A)$ and $a^{\prime} \in A$ with $a a^{\prime}-e \in \mathbf{P}(A)$ and $a-a e \in \mathbf{P}(A)$. Fix $\mathfrak{m} \in \operatorname{Max} A$ and let $x=\mathbf{B}(A) \cap \mathfrak{m}$. For $e \in x$, $e(1-e)=0$ shows that $e$ is in the kernel $K$ of the localization $R \rightarrow R^{(\mathfrak{m})}$, i.e., $x R \subseteq K$. Now consider $s \in A \backslash \mathfrak{m}$. There are $s^{\prime} \in A, e \in \mathbf{B}(A)$ and $n \in \mathbf{P}(A)$ such that $s s^{\prime}=e+n$ and $s e-s \in \mathbf{P}(A)$; it follows that $e \notin x$. Since $1+n$ is a unit in $A, s s^{\prime}(1+n)^{-1}=(e+n)(1+n)^{-1}$. The equation becomes $s s^{\prime}(1+n)^{-1}=1(\bmod x A)$. Hence, $R^{(\mathfrak{m})}$ is a homomorphic image of $R$ since the inverse of $s \in A \backslash \mathfrak{m}$ is the image of an element of $A$ modulo $K$. Hence, $R^{(\mathfrak{m})}$ is clean.

The statements for strongly clean rings are proved similarly.

(ii) This will be seen to be a special case of part (i) once it has been shown that each $R^{(\mathfrak{m})}$ is clean. Here $R^{(\mathfrak{m})}$ is a locally module finite algebra over a local one dimensional image of $A$, say $B$. Then every element of $R^{(\mathfrak{m})} / \mathbf{P}(B) R^{(\mathfrak{m})}$ is in a finite dimensional algebra over the field $B / \mathbf{P}(B)$ and, thus, is in a perfect ring. Hence, it is clean ([5, Corollary $4.2(2)])$ and, therefore, $R^{(\mathfrak{m})} / \mathbf{P}(B) R^{(\mathfrak{m})}$ is a clean ring. Moreover, since $\mathbf{P}(B) R^{(\mathfrak{m})}$ is a nil ideal idempotents lift over it and, hence, $R^{(\mathfrak{m})}$ is a clean ring (Han and Nicholson, [14, Proposition 6]), as required.

However, it is not the case that an integral extension of a commutative clean ring is necessarily a clean ring. If a prime integer $p$ is ramified in the ring of integers of an algebraic extension field $F$ of $\mathbf{Q}$ then the algebraic closure of $\mathbf{Z}_{(p)}$ in $F$ will not be local, and, hence, not a clean ring.

In Proposition 2.8 (ii), if $A$ is a regular ring then the locally module finite $A$-algebra $R$ is, in fact, a strongly clean ring.

\section{On minimal clean embeddings.}

If $R$ is embedded in a clean ring $S$, even as an essential ring extension, it is not necessarily a smallest clean extension of $R$ in $S$; for example, $\mathbf{Z}$ embedded in $\mathbf{Q}$ is essential but there are the local rings of the form $\mathbf{Z}_{\langle p\rangle}$, for every prime $p$, strictly between the two. The theme of this section is to ask when, given an embedding of $R$, not a clean ring, in a 
clean ring $S$, there are minimal clean rings between $R$ and $S$. The first step is to show that in some special cases such minimal clean extensions exist.

Recall that a local ring is any ring $R$ such that $R / \mathbf{J}(R)$ is a division ring. It is now seen that there are cases where minimal clean extensions exist.

Proposition 3.1. Let $R$ be a ring which is embedded in a local ring $S$. Then the set of clean rings between $R$ and $S$ has minimal elements.

Proof. It may be assumed that $R$ is not a clean ring. First note that a clean subring $T$ of $S$ is necessarily local. Indeed, if $a \in T$ is not a unit then, because there are no non-trivial idempotents in $T$, for any $t \in T$, $t a$ and $a t$ are also non-units. It follows that $1-a$ is a unit in $T$; hence, $a \in \mathbf{J}(R)$. Next, if $\left\{T_{\alpha}\right\}, \alpha \in A$ is a descending chain of clean (local) rings between $R$ and $S$, it is readily seen that $\bigcap_{\alpha \in A} T_{\alpha}$ is also local.

Recall that a commutative ring is a pp-ring if every principal ideal is projective. These have been characterized by Bergman ([2, Corollary 3.2]) as commutative rings $R$ where $Q=Q_{\mathrm{cl}}(R)$ is regular and $\mathbf{B}(R)=\mathbf{B}(Q)$.

Proposition 3.2. Let $R$ be a commutative ring and suppose that $S=$ $Q_{c l}(R)$ is a clean ring and that $\mathbf{B}(S)=\mathbf{B}(R)$. Then, the set of clean rings $T, R \subseteq T \subseteq S$, has minimal elements. This applies, in particular, when $R$ is a pp-ring and, more generally, if $R$ has Pierce stalks domains and $Q_{c l}(R)$ is a clean ring.

Proof. Suppose $\left\{T_{\alpha}\right\}_{\alpha \in A}$ is a descending chain of clean rings between $R$ and $S$. For $x \in \operatorname{Spec} \mathbf{B}(S)$, each $\left(T_{\alpha}\right)_{x}$ is an indecomposable clean ring, and, hence, a local ring; it follows that it is a localization of $R_{x}$ at some prime ideal $\left(P_{\alpha}\right)_{x}$, because $S_{x} \subseteq Q_{\mathrm{cl}}\left(R_{x}\right)$. Since for $\beta>\alpha$, $\left(T_{\beta}\right)_{x} \supseteq\left(T_{\alpha}\right)_{x}$, it follows that $\left(P_{\beta}\right)_{x} \subseteq\left(P_{\alpha}\right)_{x}$. For each $x$, let $P_{x}=$ $\bigcup_{\alpha \in A}\left(P_{\alpha}\right)_{x}$. Let $T=\bigcap_{\alpha \in A} T_{\alpha}$. The claim is that $T$ is clean.

Look at the stalk $T_{x}$; it needs to be shown that $T_{x}$ is local. Let $a \in T$ be such that $a_{x}$ is a unit in $T_{x}$. Then, $a_{x}$ is not in any $\left(P_{\alpha}\right)_{x}$. There is $e \notin x$ such that $b=a e+(1-e)$ is a unit in $T$ (recall that $\mathbf{B}(T)=\mathbf{B}(S))$. Hence, $b_{y} \notin P_{y}$ for any $y \in \operatorname{Spec} \mathbf{B}(T)$. Every unit in $T_{x}$ comes from a unit in $T$.

Next, if $a \in T$ is such that $a_{x} \notin P_{x}$. Then, $a_{x}$ is invertible in each $\left(T_{\alpha}\right)_{x}$ and its inverse is, thus, in each $\left(T_{\alpha}\right)_{x}$. Hence, $a_{x}$ is invertible in $T_{x}$. It follows that $T_{x}$ is local with unique maximal ideal $P_{x}$. Then, the set of clean rings between $R$ and $S$, ordered by reverse inclusion has minimal elements, by Zorn's Lemma. 
The last statement follows from [3, Proposition $2.10(1)]$, which says that if the Pierce stalks of $R$ are domains then $\mathbf{B}\left(Q_{\mathrm{cl}}(R)\right)=\mathbf{B}(R)$.

The requirement in the last statement of Proposition 3.2 that $Q_{\mathrm{cl}}(R)$ be clean cannot be dropped as [3, Example $2.9(1)]$ shows: it is a ring $R=Q_{\mathrm{cl}}(R)$ which is not clean but whose stalks are domains.

Corollary 3.3. Let $R$ be embedded in a commutative clean ring $S$ where $\mathbf{B}(S)=\mathbf{B}(R)$ and $Q_{c l}(R) \subseteq S$. Then, the set of clean rings between $R$ and $S$ has minimal elements.

Proof. The first thing to note is that if $T$ is a clean ring $R \subseteq T \subseteq S$, then $Q_{\mathrm{cl}}(R) \cap T$ is clean. Indeed, if $q \in Q_{\mathrm{cl}}(R) \cap T$ then $q=e+u$ where $e \in B(S)$ and $u$ is a unit in $T$. Hence, $u \in Q_{\mathrm{cl}}(R) \cap T$. Moreover, $u$ is a non zero-divisor in $Q_{\mathrm{cl}}(R)$ and, hence, has an inverse in both rings. Thus $u$ is a unit in the intersection. It follows, in particular, that $Q_{\mathrm{cl}}(R)$ is clean.

If, for some well-ordered set $A,\left\{T_{\alpha}\right\}_{\alpha \in A}$ is a descending chain of clean rings between $R$ and $S$, then $\left\{Q_{\mathrm{cl}}(R) \cap T_{\alpha}\right\}_{\alpha \in A}$ is a descending chain of clean rings in $Q_{\mathrm{cl}}(R)$. Now Proposition 3.2 applies to this chain giving a clean ring which is a lower bound for the original chain.

If $R$ is any commutative ring which is embedded in a commutative regular ring $S$ then the corollary applies to $R[\mathbf{B}(S)]$ and $S$.

Recall that a $p m$ ring $R$ is such that for every prime ideal $\mathfrak{p}$ of $R$ there is a unique maximal ideal $\mathfrak{m}$ with $\mathfrak{p} \subseteq \mathfrak{m}$. Moreover, a ring $R$ such that every $r \in R$ can be expressed $r=e+s, e^{2}=e$ and $s$ a non zero-divisor, is called an almost clean ring. Every commutative clean ring is a pm ring ([1, Corollary 4$])$.

The rings $R$ in Proposition 3.2, it should be noted, are not pm rings.

Proposition 3.4. Let $R$ be a commutative pm ring. If $R$ is embedded in a commutative clean ring $S$ then the subring $T$ of $S$ generated by $R$ and $\mathbf{B}(S)$ is clean.

Proof. According to [3, Theorem 2.4], a pm ring which is almost clean is clean and, moreover, an extension of a pm ring by idempotents is again a pm ring ([3, Corollary 1.6]). Hence, it suffices to show that $T$ is an almost clean ring. This is immediate: if $t \in T$ then $t=\sum_{i=1}^{n} r_{i} e_{i}$, where each $r_{i} \in R$ and $\left\{e_{1}, \ldots, e_{n}\right\}$ is a complete orthogonal set of idempotents from $\mathbf{B}(T)=\mathbf{B}(S)$. Write $r_{i}=f_{i}+u_{i}, i=1, \ldots n$, $f_{i}^{2}=f_{i}$ and $u_{i}$ a unit in $S$. Then, $t=\sum_{i=1}^{n} f_{i} e_{i}+\sum_{i=1}^{n} u_{i} e_{i}$; the first term is an idempotent and the second, which is in $T$, is a unit in $S$ and, hence, a non zero-divisor in $T$. 
Notice, in the proposition, that the Pierce stalks of the ring $R[\mathbf{B}(S)]$ are local homomorphic images of $R$.

The next result is to show that if $R$ is a commutative pm ring which is not clean is embedded in a commutative clean ring $S$, there is no clean ring $T, R \subseteq T \subseteq S$ which is minimal with that property.

The following topological lemma, kindly supplied to us by R.G. Woods, will be used. The proof will appear in an appendix.

Lemma 3.5. Let $X$ be a compact Hausdorff space which is not a boolean space. Suppose $Y$ is a boolean space and that there is a continuous surjection $\sigma: Y \rightarrow X$. Then, there are $y_{1} \neq y_{2}$ in $Y$ with $\sigma\left(y_{1}\right)=\sigma\left(y_{2}\right)$ and $(i)$ the quotient space $Z=Y /\left\{y_{1}, y_{2}\right\}$ is a boolean space, (ii) $\sigma$ factors through $Z, \sigma=\tau^{\prime} \circ \tau$, and (iii) $\tau^{\prime}$ is not a homeomorphism.

The key point in the proof is that if, in $X$, two elements are sent to the same image under $\sigma$, they can be identified and the new quotient space remains a boolean space.

Properties of rings of continuous functions on Tychonoff spaces suggested the following result.

Theorem 3.6. Let $R$ be a commutative pm ring which is not a clean ring. If $S$ is a commutative clean ring $R \subseteq S$, then there is a clean ring $T$ strictly between $R$ and $S$.

Proof. If $S \neq R[\mathbf{B}(S)]$, then, by Theorem 3.4, the theorem is proved. Otherwise, it may be assumed that $S=R[\mathbf{B}(S)]$. By $\mathrm{Lu}$ and $\mathrm{Yu}$, [19, Corollary 2.7], $\operatorname{Max} R$ is not zero-dimensional, i.e., the compact Hausdorff space Max $R$ is not a boolean space. However, Max $S$ and $\operatorname{Spec} \mathbf{B}(S)$ are homeomorphic and are boolean spaces. There is a continuous surjection $\operatorname{Max} S \rightarrow \operatorname{Max} R$. Indeed, let $\phi: \operatorname{Max} S \rightarrow \operatorname{Spec} R$ be the function given by intersection and $\Theta_{R}$ : Spec $R \rightarrow \operatorname{Max} R$ the continuous surjection available in pm rings (De Marco and Orsatti $[8$, Theorem 1.2]) where $\Theta_{R}(\mathfrak{p})$ is the unique maximal ideal containing $\mathfrak{p}$. For every minimal prime ideal $\mathfrak{p}$ of $R, \mathfrak{p} S$ is a proper ideal. Hence, for every minimal prime ideal $\mathfrak{p}$ of $R$, the image of $\phi$ contains a prime ideal containing $\mathfrak{p}$. Thus, $\psi=\Theta_{R} \circ \phi$ is the desired continuous surjection. Since Max $S$ is a boolean space and $\operatorname{Max} R$ is compact and Hausdorff but not a boolean space, $\psi$ cannot be one-to-one. According to Lemma 3.5, $\psi$ factors $\psi=\tau \circ \sigma$,

$$
\operatorname{Max} S \stackrel{\sigma}{\rightarrow} Y \stackrel{\tau}{\rightarrow} \operatorname{Max} R,
$$

where $Y$ is a boolean space, $\sigma$ is a surjection and $\tau$ is not one-to-one. 
It is now temporarily assumed that $S$ is semiprime. The plan is to construct a Pierce sheaf over $Y$ of a clean ring $T$ strictly between $R$ and $S$. Suppose that when using Lemma $3.5, \mathfrak{m}_{1}$ and $\mathfrak{m}_{2}$ are distinct elements of $\operatorname{Max} S$ with the same image $\mathfrak{n}$ under $\psi$ and that these have been identified in forming $Y$. The Pierce stalks of $S$ corresponding to $\mathfrak{m}_{1}$ and $\mathfrak{m}_{2}$ are denoted $S_{u_{1}}$ and $S_{u_{2}}$ while the corresponding point of $Y$ is called $y$. The rings $S_{u_{i}}, i=1,2$, are local homomorphic images of $R$, say, $R / I_{i}$. The ideals $I_{i}$ are semiprime, by the assumption, and the only maximal ideal containing each is $\mathfrak{n}$. According to [8, Theorem 1.2], the intersection of all the prime ideals contained in $\mathfrak{n}, \mathcal{O}_{\mathfrak{n}}$, is contained in only one maximal ideal, namely $\mathfrak{n}$. Hence, $I_{1} \cap I_{2}$ is a semiprime ideal contained in only one maximal ideal. Thus, $R /\left(I_{1} \cap I_{2}\right)$ is local.

The sheaf construction can now proceed. For $z \in Y, z \neq y, z$ can be identified with a stalk of $S$ which can be called $S_{z}$ and $T_{z}=S_{z}$; the stalk over $y$ will be $T_{y}=R /\left(I_{1} \cap I_{2}\right)$. Notice that a clopen (closed and open) set in $Y$ lifts to a clopen set of $\operatorname{Spec} \mathbf{B}(S)$ which either contains or excludes both $u_{1}$ and $u_{2}$. A topology will be introduced in the disjoint union of the stalks, $\mathcal{T}$, and it will need to be verified that the new structure satisfies the conditions of a reduced ringed space ([25, Definitions 3.1 and 4.1]). Basic neighbourhoods in $\mathcal{T}$ are defined as follows: each $T_{z}$ is a local ring of the form $R / I(z)$ and, for a fixed $z \in Y$ consider a point $r+I(z)$. Let $N$ be a clopen set of $Y$ and define $\{r+I(v) \mid v \in N\}$ to be an open set in $\mathcal{T}$. The key thing to verify is that if $r+I(z)=s+I(z)$ for $r, s \in R$ and some $z \in Y$, there needs to be a neighbourhood $N$ of $z$ over which the equality holds. If $z \neq y$, there is a clopen set $N$ in $\operatorname{Spec} \mathbf{B}(S)$, which may be taken to exclude $u_{1}$ and $u_{2}$ such that $r+I(v)=s+I(v)$, for all $v \in N$. Since $N$ may also be viewed as a clopen set in $Y$, this case has been dealt with. When $r+I(y)=s+I(y)$ then $r-s \in I(y)=I_{1} \cap I_{2}$; then, there are clopen neighbourhoods $N_{i}, i=1,2$, in $\operatorname{Max} S$, so that $r+I(v)=s+I(v)$ for all $v \in N_{1}$ and for all $v \in N_{2}$. Then, $N_{1} \cup N_{2}$ becomes, via $\sigma$, a neighbourhood $N$ of $y$ in $Y$. This completes the verification.

The ring of sections of this sheaf, call it $T$, is a clean ring because its stalks are local. Since $\bigcap_{z \in Y} I(z)=\mathbf{0}, T$ extends $R$ and lies in $S$ since any element of $T$ yields a section in the sheaf for $S$ by replacing $r+I(y)$ with $r+I_{1}$ and $r+I_{2}$. Finally, $T$ is strictly between $R$ and $S$ because $T$ is clean while $R$ is not, and any idempotent of $S$ whose support contains exactly one of $u_{1}$ or $u_{2}$ cannot be in $T$.

The assumption that $S$ be semiprime is now dropped. Consider the rings $\bar{R}=R / \mathbf{P}(R)$ and $\bar{S}=S / \mathbf{P}(S)$. Clearly $\bar{R}$ is a pm ring which is not clean and $\bar{S}$ is a semiprime clean ring. By the above reasoning, there is a clean ring $\bar{T}$ strictly between $\bar{R}$ and $\bar{S}$. Set $T=\{t \in S \mid$ 
$t+\mathbf{P}(S) \in \bar{T}\}$. Since $T / \mathbf{P}(T)=\bar{T}, T$ is a clean ring which is strictly between $R$ and $S$.

Corollary 3.7. If $R$ is a commutative pm ring, which is not a clean ring, embedded in a commutative clean ring $S$, then there is no clean ring between $R$ and $S$ which is minimal with the property of being a clean ring and lying between $R$ and $S$.

The corollary applies, in particular, to any ring of continuous real valued function $C(X)$, where $X$ is a Tychonoff space which is not strongly zero-dimensional (i.e., $\beta X$ is not a boolean space, McGovern, [20, Theorem 13]) embedded in its complete ring of quotients. When $X$ is a metric space which is not strongly zero-dimensional, $Q_{\mathrm{cl}}(C(X))$ is a regular ring and, by Corollary 3.7, there are no minimal clean rings between $C(X)$ and $Q_{\mathrm{cl}}(C(X))$; this is in contrast to Corollary 3.3.

If $X$ is a compact Hausdorff space which is not a boolean space, the ring $C(X)$ is not clean; when $C(X)$ is embedded in a commutative clean ring (e.g. $Q(X))$ then there is a continuous surjection $Y=\operatorname{Max} S \rightarrow$ $X$. This gives rise to an embedding $C(X) \subseteq C(Y), C(Y)$ is a clean ring. Lemma 3.5 can then be used to get countable chains of boolean spaces connected by continuous surjections $Y \rightarrow Y_{1} \rightarrow Y_{2} \rightarrow \cdots \rightarrow X$ and then chains of clean extension rings of $C(X), C(X) \subseteq \cdots C\left(Y_{2}\right) \subseteq$ $C\left(Y_{1}\right) \subseteq C(Y)$.

There is a special kind of clean ring called uniquely clean where each element is expressible as a sum of an idempotent and a unit but in exactly one way. These are characterized (Nicholson and Zhu [24, Theorem 20]) as rings $R$ where $R / \mathbf{J}(R)$ is a boolean ring and idempotents lift modulo $\mathbf{J}(R)$. The behaviour here is different from what has gone before.

Proposition 3.8. Let $R$ be a ring embedded in a uniquely clean ring $S$. Then, there is a unique minimal clean ring between $R$ and $S$, and all the clean rings between $R$ and $S$ are uniquely clean.

Proof. That all the clean rings between $R$ and $S$ are uniquely clean is obvious. If $T$ is the intersection of all clean rings between $R$ and $S$, for $t \in T, t=e+u \in S, e=e^{2}$ and $u$ a unit, has the same expression in all the rings of the intersection.

By [24, Corollary 10 and Proposition 13], if $R$ is a uniquely clean ring then $R[[X]]$ is uniquely clean but $R[X]$ is not. Proposition 3.8 applies to this situation. When $R=\mathbf{Z}_{2}$, Proposition 3.7 can also be used and, in fact, the unique minimal clean ring between $\mathbf{Z}_{2}[X]$ and $\mathbf{Z}_{2}[[X]]$ is $\mathbf{Z}_{2}[X]_{(X)}$. It can be seen that, even with respect to homomorphisms to 
uniquely clean rings, that $\mathbf{Z}_{2}[X]$ in $\mathbf{Z}_{2}[X]_{(X)}$ does not satisfy a universal property.

The following simple remark fits in with theme of this section.

Remark 3.9. Let $R$ be a clean ring embedded in a ring $S$. Then there are clean rings between $R$ and $S$ maximal with the property of being a clean ring.

Proof. It only needs to be observed that the union of a chain of clean rings is again a clean ring.

\section{More on clean extensions of commutative pm rings.}

The embedding of a commutative ring $R$ in a commutative clean ring $S$ cannot be such that $S_{R}$ is finitely generated unless $R$ is a pm ring, indeed, by [3, Proposition 3.11], no integral extension of $R$ can be clean when $R$ is not a pm ring. However, when $R$ is a commutative pm ring there is always an integral extension which is a clean ring, even one generated by idempotents (Proposition 3.4). The next step will be to show that it is not possible to go from a pm ring $R$ which is not clean to a clean ring by the adjunction of a finite number of central idempotents.

Lemma 4.1. Let $X \neq \emptyset$ be a compact Hausdorff space. If $X$ is the union of a finite number of subspaces which are boolean spaces then $X$ contains a non-empty clopen subspace which is boolean.

Proof. Let the boolean subspaces be $V_{1}, \ldots, V_{n}$. If any one is all of $X$, there is nothing to prove. Otherwise, renumbering if necessary, assume that $W=V_{1} \cup \cdots \cup V_{k} \neq X$ but $V_{1} \cup \cdots \cup V_{k}=X$. Then, $X \backslash W$ is open both in $X$ and in the subspace $V_{k}$. Therefore, there is $\emptyset \neq U \subseteq X \backslash W$ which is clopen in $V_{k}$. Since it is compact, it is closed in $X$ and also open in $X$.

Lemma 4.2. Let $R$ be a commutative $p m$ ring and $S=R\left[e_{1}, \ldots, e_{n}\right]$ an extension of $R$ generated by a finite set of central idempotents. If $S$ is a clean ring then $R$ has a non-trivial idempotent $f$ such that $R f$ is a clean ring.

Proof. For convenience of notation, the proof will be done for $n=2$. It first must be noted how maximal ideals behave in the two rings. First, $S=S e_{1} e_{2}+S e_{1}\left(1-e_{2}\right)+S\left(1-e_{1}\right) e_{2}+S\left(1-e_{1}\right)\left(1-e_{2}\right)=$ $R e_{1} e_{2}+R e_{1}\left(1-e_{2}\right)+R\left(1-e_{1}\right) e_{2}+R\left(1-e_{1}\right)\left(1-e_{2}\right)$. (In the general case there will be $2 \cdot n$ ! terms.) Since the extension is integral, contraction $\phi: \operatorname{Max} S \rightarrow \operatorname{Max} R$ is a surjection. Give $\mathfrak{n} \in \operatorname{Max} S$, either $e_{i} \in \mathfrak{n}$ or $1-e_{i} \in \mathfrak{n}, i=1,2$. Hence, of the four terms listed above, three must 
be in $\mathfrak{n}$, say, $R e_{1} e_{2}+R e_{1}\left(1-e_{2}\right)+R\left(1-e_{1}\right) e_{2} \subseteq \mathfrak{n}$. It follows readily that there is a unique $\mathfrak{m} \in \operatorname{Max} R$ such that $\mathfrak{n}=\operatorname{Re}_{1} e_{2}+\operatorname{Re}_{1}\left(1-e_{2}\right)+$ $R\left(1-e_{1}\right) e_{2}+\mathfrak{m}\left(1-e_{1}\right)\left(1-e_{2}\right)$, and, similarly, for the other possible combinations.

It is seen that Max $S$ splits into four disjoint subspaces, depending on which of $e_{1}, e_{2}$ lies in a maximal ideal. These can be identified with $W_{1}=\operatorname{Max} R e_{1} e_{2}, W_{2}=\operatorname{Max} R e_{1}\left(1-e_{2}\right), W_{3}=\operatorname{Max} R(1-$ $\left.e_{1}\right) e_{2}$ and $W_{4}=\operatorname{Max} R\left(1-e_{1}\right)\left(1-e_{2}\right)$, respectively, and are, hence, disjoint boolean subspaces of Max $S$. Moreover, the description of the maximal ideals of $S$ shows that $\phi$ restricted to each $W_{i}$ is injective. Let $\phi\left(W_{i}\right)=V_{i} \subseteq \operatorname{Max} R$. Since $W_{i}$ is compact and $\operatorname{Max} R$ is Hausdorff, $V_{i}$ is compact and as a result, $\left.\phi\right|_{W_{i}}: W_{i} \rightarrow V_{i}$ is a homeomorphism.

Now Lemma 4.1 applies to the $V_{i}$ in Max $R$ showing that there exists $\emptyset \neq U \subseteq \operatorname{Max} R$ which is a boolean subspace. In a pm ring there is a continuous surjection $\Theta_{R}: \operatorname{Spec} R \rightarrow \operatorname{Max} R$ ([8, Theorem 1.2]) and, hence, $\Theta_{R}^{-1}(U)$ is a clopen set of $\operatorname{Spec} R$ which corresponds to some $f \in \mathbf{B}(R)$. It is readily seen that $R f$, as a ring, is pm and Max $R f=U$. Hence, $R f$ is a clean ring ([19, Theorem 2.6]).

Theorem 4.3. Let $R$ be a commutative $p m$ ring which is not a clean ring and $S$ an extension ring of the form $S=R\left[e_{1}, \ldots, e_{n}\right]$ for some $e_{1}, \ldots, e_{n} \in \mathbf{B}(S)$. Then, $S$ is not a clean ring.

Proof. By Lemma 4.2 it would suffice if the proof could be reduced to the case where $\mathbf{B}(R)$ is trivial. Since $R$ is clean if and only if each of its Pierce stalks is clean (i.e., local), there is some $x \in \operatorname{Spec} \mathbf{B}(R)$ where $R_{x}=R / R x$ is not clean. However, $\mathbf{B}\left(R_{x}\right)$ is trivial. Now consider $T=S / S x$; this is not a Pierce stalk of $S$ but is a factor ring which is clean if $S$ is clean. Now write $T=R_{x}\left[\overline{e_{1}}, \ldots, \overline{e_{n}}\right]$, where $\overline{e_{i}}$ is the image of $e_{i}$ in $T, i=1, \ldots, n$. Note that $R_{x}$ embeds in $T$ since, for $r \in R$, if $r \in S x, r$ can be written (again simplifying to the case of $n=2) r=r_{1} e_{1} e_{2}+r_{2} e_{1}\left(1-e_{2}\right)+r_{3}\left(1-e_{1}\right) e_{2}+r_{4}\left(1-e_{1}\right)\left(1-e_{2}\right)$ with $r_{i} \in R x$. It follows that there exist $f_{i} \in x$ such that $r_{i}=r_{i} f_{i}$, $i=1, \ldots, 4$. The supremum $f$ of these idempotents is also in $x$. Hence, $r_{i}=r_{i} f \in R x, i=1, \ldots, 4$, yielding $r=r f \in R x$. Therefore, the kernel of $R_{x} \rightarrow R_{x}[\bar{e}]=T$ is trivial. Lemma 4.2 now says that $T$ is not clean, hence, that $S$ is not clean.

The finite integral extension in Theorem 4.3 is of a special kind. It is not clear whether other sorts of finite integral extensions can lead to a clean ring. Various reductions are possible and it may be assumed that $R$ is indecomposable and that $R[a]$ is a clean ring for a single integral element $a$. The problem is posed formally. 
Question 4.4. Let $R$ be a commutative pm ring which is not a clean ring. Can there exist an extension ring $S$ which is a clean ring and the module ${ }_{R} S$ is finitely generated?

The following example, a variant of one kindly supplied by G.M. Bergman, does not answer the question but it does show how adding one idempotent or an integral unit to an indecomposable pm ring can produce uncountably many new idempotents.

Example 4.5. Let $C=C([0,1])$ and $R$ the subring of $\Pi=\prod_{\mathbf{N}} C$ defined by $\left\{\left(c_{n}\right) \in \Pi \mid c_{2 n}(1 / 2)=c_{2 n+1}(1 / 3)\right.$ and $\left.c_{2 n+1}(1 / 3)=c_{2 n+2}(1 / 4)\right\}$. Then, $R$ is an indecomposable pm ring. If $e \in \Pi$ is such that $e_{2 n}=1$ and $e_{2 n+1}=0$ for $n \in \mathbf{N}$ and $u \in \Pi$ is such that $u_{2 n}=1$ and $u_{2 n+1}=-1$ then $R[e]=R[u]=\Pi$.

Proof. First note that $R$ is indecomposable. Any idempotent $f$ must have components 0 or 1 for each $n \in \mathbf{N}$. If $f$ is non-trivial it cannot be in $R$. Next, assuming for the moment that $R[e]=\Pi, R$ must be a pm ring since, by [3, Theorem 1.2], an integral extension of a ring not a pm ring cannot be a pm ring and, moreover, a product of pm rings, such as $\Pi$, is a pm ring ([7, Theorem 3.3]). The subset $R e$ contains all elements of $\Pi$ with support in the even integers and, similarly, $R(1-e)$ has all those with support in the odds. Hence, $R[e]=\Pi$. Finally, $\frac{1}{2}(u+1)=e$.

5. Appendix - proof of Lemma $3.4([27])$. Let $X$ be a compact Hausdorff space which is not a boolean space and suppose $Y$ is a boolean space and that there is a continuous surjection $\sigma: Y \rightarrow X$. The following facts will be used. (1) If $W$ is a locally compact zerodimensional space (there is a basis of clopen sets), then the one-point compactification $\omega W$ is a boolean space. (2) A continuous bijection from one compact Hausdorff space to another is a homeomorphism. (3) If $Y$ is a boolean space and $F \subseteq Y$ is a finite subset, the quotient space $Y / F$ is again a boolean space, in fact it is homeomorphic to $\omega(Y \backslash F)$.

With these facts in hand, if $\sigma: Y \rightarrow X$ is a continuous surjection where $Y$ is a boolean space and $X$ is a compact Hausdorff space which is not boolean, then $\sigma$ cannot be one-to-one and there is some $x \in X$ where $\left|\sigma^{-1}(x)\right|>1$. Take $y_{1} \neq y_{2}$ in $\sigma^{-1}(x)$ and form, as above, the quotient space formed by identifying $y_{1}$ and $y_{2}$ and call the new boolean space $Z$ with $z \in Z$ the common image of $y_{1}$ and $y_{2}$. By the property of a quotient space, $\sigma$ factors through $Z$, say $Y \stackrel{\tau}{\rightarrow} Z \stackrel{\tau^{\prime}}{\rightarrow} X$. Since $Z$ is a boolean space and $X$ is not, $\tau^{\prime}$ cannot be one-to-one. 


\section{REFERENCES}

[1] D.D. Anderson and V.P. Camillo, Commutative rings whose elements are a sum of a unit and idempotent, Comm. Algebra 30 (2002), 3327-3336.

[2] G. Bergman, Hereditary commutative rings and centres of hereditary rings, Proc. London Math. Soc. 23 (1971), 214-236.

[3] W.D. Burgess and R. Raphael, On commutative clean rings and pm rings, Comtemp. Math. 480 (2009) 35 - 55.

[4] W.D. Burgess and W. Stephenson, Rings all of whose Pierce stalks are local, Canad. Math. Bull. 22 (1979), 159-164.

[5] V.P. Camillo, D. Khurana, T.Y. Lam, W.K. Nicholson and Y. Zhou, Continuous modules are clean, J. Algebra 304 (2006), 94-111.

[6] V.P. Camillo and H.-P. Yu, Exchange rings, units and idempotents, Comm. Algebra 22 (1994), 4737-4749.

[7] M. Contessa, On PM-rings, Comm. Algebra 10 (1982), 93-108.

[8] G. De Marco and A. Orsatti, Commutative rings in which every prime ideal is contained in a unique maximal ideal, Proc. Amer. Math. Soc. 30 (1971), 459-466.

[9] S. Feigelstock, Additive Groups of Rings, Volume 1. Pitman Research Notes in Mathematics, 83, Longman, 1983.

[10] S. Feigelstock, Additive Groups of Rings, Volume II. Pitman Research Notes in Mathematics, 169, Longman, 1988.

[11] N.J. Fine, L. Gillman and J. Lambek, Rings of Quotients of Rings of Functions. Lecture Notes in Real Algebraic and Analytic Geometry, RAAG (2005).

[12] L. Gillman and M. Jerison, Rings of Continuous Functions. Grad. Texts in Math. 43, Springer-Verlag, Berlin, 1976.

[13] K.R. Goodearl and R.B. Warfield, Jr., Algebras over zero-dimensional rings, Math. Ann. 223 (1976), 157-168.

[14] J. Han and W.K. Nicholson, Extensions of clean rings, Comm. Algebra 29 (2001), 2589-2595.

[15] B. Huisgen-Zimmermann, Purity, algebraic compactness, direct sum decompositions, and representation type, in Infinite Length Modules, Trends in Mathematics, Birkhäuser, 2000.

[16] P.T. Johnstone, Stone Spaces. Cambridge Studies in Advanced Mathematics 3 Cambridge University Press, Cambridge 1992.

[17] C.U. Jensen and B. Zimmermann-Huisgen, Algebraic compactness of ultrapowers and representation type, Pacific J. Math. 139 (1989), 251-265.

[18] T.Y. Lam, Lectures on Modules and Rings. Graduate Texts in Mathematics 189, Springer-Verlag, 1998.

[19] D.C. $\mathrm{Lu}$ and W.H. Yu, On prime spectrums of commutative rings, Comm. Algebra 34 (2006), 2667-2672.

[20] W.Wm. McGovern, Clean semiprime $f$-rings with bounded inversion, Comm. Algebra 31 (2003), 3295-3304.

[21] S.H. Mohamed and B.J. Müller, Continuous and Discrete Modules. London Math. Soc. Lecture Note Series 147, Cambridge 1990.

[22] W.K. Nicholson, K. Varadarajan and Y. Zhou, Clean endomorphism rings, Arch. Math. 83 (2004), 340-342.

[23] W.K. Nicholson, Lifting idempotents and exchange rings, Trans. Amer. Math. Soc. 229 (1977), 269-278. 
[24] W.K. Nicholson and Y. Zhou, Rings in which elements are uniquely the sum of an idempotent and a unit, Glasg. Math. J. 46 (2004), 227-236.

[25] R.S. Pierce, Modules over Commutative Regular Rings. Mem. Amer. Math. Soc. 70, Providence, RI, 1967.

[26] Z. Wang and J.L. Chen, 2-clean rings, Canad. Math. Bull. 52 (2009), 145153.

[27] R.G. Woods, private communication (2010).

Department of Mathematics and Statistics, University of Ottawa, OtTaWa, ON, CANAdA K1N 6N5

E-mail address: wburgess@uottawa.ca

Department of Mathematics, Concordia University, Montreal, QC, CANADA H4B 1R6

E-mail address: raphael@alcor.concordia.ca 